\title{
Wide Area Measurement System in the IEEE-14 Bus System using Multiobjective Shortest Path Algorithm for Fault Analysis
}

\author{
Lilik J. Awalin ${ }^{1 *}$, Syahirah Abd Halim², Jafferi Bin Jamaludin ${ }^{3}$, Nor Azuana Ramli ${ }^{4}$ \\ Faculty of Advanced Technology and Multidiscipline, Airlangga University ${ }^{1}$ \\ Gedung Kuliah Bersama, Kampus C Unair, Jl. Mulyorejo, Surabaya 60155, Indonesia ${ }^{1}$ \\ Department of Electrical, Electronic \& Systems Engineering, Faculty of Engineering \& Built Environment ${ }^{2}$ \\ Universiti Kebangsaan Malaysia, Selangor, Malaysia ${ }^{2}$ \\ UM Power Energy Dedicated Advanced Centre (UMPEDAC), Kuala Lumpur, Malaysia ${ }^{3}$ \\ Centre For Mathematical Sciences, Universiti Malaysia Pahang),Gambang, Pahang, Malaysia ${ }^{4}$
}

\begin{abstract}
In a large-scale interconnected power system network, few challenges exist in evaluating and maintaining the overall system stability. The power system's ability to supply all types of loads during natural disasters or faults has yet to be addressed. This work focuses on developing a wide-area measurement system to manage and control the power system under all operating conditions. The IEEE-14 bus system was modeled in PSCAD software for simulating nineteen types of fault based on multi-objective shortest path algorithm. To manage the wide area measurement, the research must comprehend the working principle of the multi-objective shortest path algorithm, whereby the proposed method will determine the new path for the IEEE-14 bus system. To evaluate the performance of the multi-objective shortest path algorithm, all sections of the IEEE-14 bus system were simulated with faults. The distances of the normal path (without simulated fault) and the new path (with simulated fault) were recorded. Based on the recorded data, it was found that the location of the fault has significant influence on the shortest path of the buses connected to each other.
\end{abstract}

Keywords-IEEE-14 bus system; wide area measurement; multi-objective shortest path algorithm; fault location; PMU

\section{INTRODUCTION}

SCADA system used for real-time monitoring of a power system network is not alone sufficient to provide data related to dynamic performance of the overall system in steady state condition. The actual performance of the system can be predicted by utilising any power system simulation tools, which normally have few limitations in term of parameters exactness and generalized estimation. Therefore, the system operator must be ensuring of consistent and detailed dynamic information in order to decide on how to deal with the system during specific conditions and energy security.

The technique is based on close observation on the angle and phasors with respect to time duration in dynamic state by using phasor measurement unit (PMU). PMU will show the dynamic information of individual buses in the system for the system operator. This information will be useful in determining how to respond to the system's condition in a timely manner. The PMU can also be used as an investigator apparatus to monitor performance of hardware in the system, such as generator controller. Additionally, the system operator needs to utilize the PMU in managing a wide area monitoring system as the one of the approaches in smart grid operation to provide the required data.

The operator must also understand the overall system behavior during dynamic phenomena based on the data obtained from the wide area monitoring system. The PMU, which is part of the wide area monitoring system, normally consists of standard components such as communication system, data analyst and information display. Placement of the PMU in the power system is dependent on a preliminary study of the system's constraints and stability problems that might possibly occur in the wide area monitoring system.

In [1], the system operator also supervises any event in the system for the purpose of monitoring and analyzing the dynamic behavior of the power system during different scenarios. The system protection, dependability and stability will be considered as well in the development process of the wide area monitoring system for the power grid. This demonstrates the significance of the wide area monitoring system in ensuring reliable operation of the power grid and improved system security. In return, this approach can help to improve the overall technical aspects by optimising the stability of the overall system and reducing the operational cost.

\section{LITERATURE REVIEW}

\section{A. Monitoring System}

An electric power system is made up of a number of interconnected components that serve a purpose of delivering reliable power supply at a low cost. The majority of the world's population is already reliant on electrical energy for a better quality of life and economic growth. However, the power system is always vulnerable to internal and external disturbances that can disrupt the system operational capability,

*Corresponding Author 
such as lightning, electromagnetic interference, and equipment malfunctions.

However, in a very large, interconnected power system, several challenges exist in assessing and maintaining the stability of the entire system as mention in [2]. Another issue in the power system related to large penetration of renewable energy sources has recently aroused, contributing to additional risk on the stability which requires extensive monitoring on the system operation. However, the development of renewable energy sources is critical for energy security. Consequently, power system monitoring should be a key aspect in accomplishing adaptable tasks to maintain system dependability and reliability of electricity supply.

In recent years, advancements in information and communication technology have enabled greater adaptability in wide-area power system monitoring in terms of rapid and massive data transmission. As one of the smart grid innovations in the power grid, the wide-area monitoring system with phasor measurement units is a promising solution towards the improvement of overall system operation, as mention in [3].

\section{B. Optimal Placement of PMU using Integer Linear Programming}

Power engineers are becoming interested in phasor measurement units (PMU) since they can provide synchronized estimates of real-time phasors of voltage and current. Furthermore, the use of PMUs has also changed the way state estimation is being performed. A state estimator plays a vital role in the security of power system operations as it is used for online monitoring, analysis and control.

According to [4], an ideal PMU placement algorithm was developed to recover the bad data processing capability of state estimation by taking advantage of the PMU technology. Techniques for identifying placement sites for phasor measurement units in a power system based on incomplete observability are presented in [5], where simulated annealing method was used to solve the pragmatic communicationconstrained in PMU placement problem. The authors in [6][10] developed an optimal placement algorithm for PMUs by using integer programming. However, the proposed integer programming becomes a nonlinear integer programming under the existence of conventional power flow or power injection measurements. Besides, a similar formulation of optimal PMU placement problem is proposed by integer linear programming. According to [11]-[12], there is two proposed formulation which is without conventional measurements and with conventional measurements. Referring to [11], simulation results show that the proposed algorithm demonstrates computational efficiency and can be used in actual practical system. However, the simulation was only performed on the IEEE-14 bus system due to computational limitations.

\section{Dynamic Shortest Path Algorithm}

Wide area monitoring system normally consists of three main elements, namely management, measurement, and communication. It is critical to plan these elements carefully for a power system to function well. According to [13]-[15], measurement and communication elements in a wide area network are planned flexibly from an administrative standpoint to achieve a suitable degree of system monitoring.

The optimal placement of phasor measurement units is determined using an integer linear programming (ILP) layout technique that takes zero-injection bus effects into consideration. The PMU location problem is solved using the integer linear programming (ILP) technique with and without conventional estimations, as well as maximum estimation redundancy across all buses. According to [16]-[19], a new decision on discernibleness limitations was proposed to address the optimal positioning issue, which in turn might reduce the number of required PMUs.

According to publications [20]-[21], a new decision method based on identifiable limits that could reduce the number of PMUs has been addressed to solve the optimal PMU position problem.

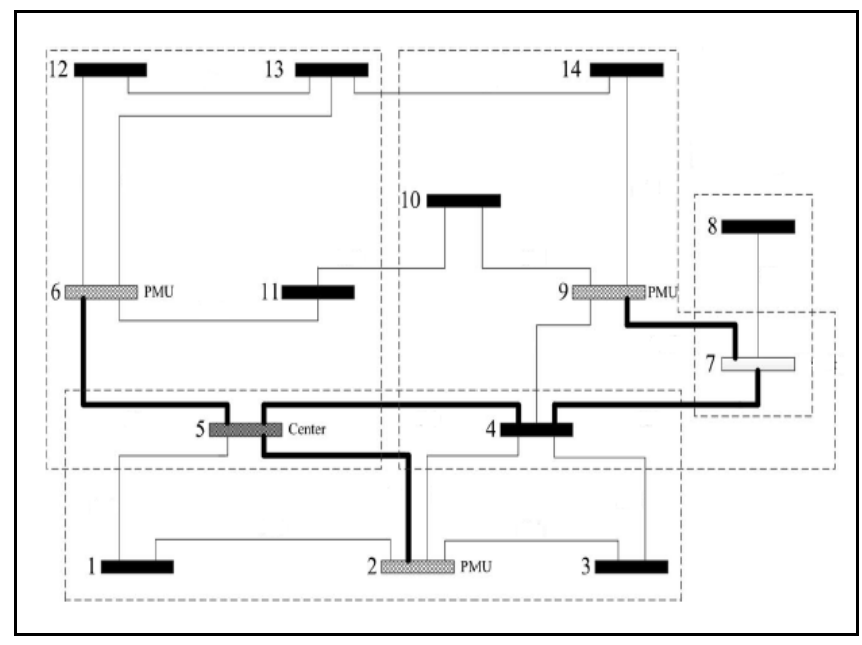

Fig. 1. Optimal Design of Wide Area Monitoring System for the IEEE 14 Bus System [22].

In [22], the PMU placement was designed as illustrated in Fig. 1. For the optimal structure of the communication framework, a dynamic multi-objective shortest path (MOSP) routing technique was implemented. As a result, the proposed technique optimizes PMU location initially, then enhances communication methods that connect all phasor measurement units. In the MOSP steering algorithm, which is used for optimal communication interface layout, the shortest path exploration algorithm is used to select the shortest pathways from phasor measurement units' buses to the central control bus. As a result, all the evaluated PMU nodes in set S carried by author in [22] may be measured as original nodes. It was also discovered that, of all the possible routes, the nearest phasor measurement units' bus has consistently the shortest route to the central control bus.

There are two ways of acquiring transmission path information from the phasor measurement units' bus to central control bus, according to [22]; single objective shortest path and multi objective shortest path. The shortest path from each single objective shortest path bus to the central control bus can be obtained directly using a shortest path algorithm. Meanwhile, in a multi objective shortest path algorithm direct 
calculation, overlapped ways are used to obtain transmission path information for all phasor measurement units' buses. Therefore, all phasor measurement units' buses, except for the closest one to the $\mathrm{CB}$, can be determined using the multi objective shortest path algorithm, owing to the overlapped ways that can be used as a method for information transmission with reduced optical fiber ground wire coverage.

\section{METHODOLOGY}

Typically, a wide area monitoring system is made up of three components: management, measurement, and communication. It is necessary to build these infrastructures adequately for the optimal operation of the power system. To achieve a satisfactory level of system observability, the measurement and communication infrastructures in a wide area network are planned independently from a management perspective. There have been numerous procedures taken in order to develop the 14-bus test system for this study. In the first step, integer linear programming was chosen to determine the optimal configuration of measuring mechanisms [22]. The dynamic multi-objective shortest path was used in the next step to come up with the best communication infrastructure option. The fault cases were assigned to monitor the performance of the multi-objective shortest path, in which 19 fault cases were simulated. For each case, the distance calculated using the multi-objective shortest route technique was measured and compared.

The flow chart involved in implementing the proposed technique is depicted in Fig. 2. The initial work involves modelling in PSCAD software to develop the IEEE 14-bus network as the test system. The PMU's position was then determined using integer linear programming (ILP). According to [11], the placement of PMUs is solved using binary integer programming in MATLAB. However, due to research limitations, this study did not develop the algorithm for PMU placement and instead relied on [22] for PMU placement using ILP on the IEEE-14 bus system.

To solve the optimum phasor measurement unit location problem, three PMU will be installed in buses 2, 6, and 9. In order to observe the performance of the multiobjective shortest path algorithm, all of the section was assigned by fault. The location of fault is listed in Table I as follows.

The following step is to assign different 19 fault cases at IEEE-14 bus system. To test the performance of the multiobjective shortest route method, each fault was allocated to various places. Fig. 3 shows the location of every fault case that was allocated in the IEEE-14 bus system. Each fault case was simulated using MATLAB software. For each fault condition, the algorithm was modified to evaluate and choose the shortest path. The result will show the distance between each fault condition and other buses. Every distance recorded by each fault case to connect to the other bus using the shortest path was then determined. Data from the 19 failure cases were recorded, and the graphs were plotted to acquire more information regarding the multi-objective shortest route algorithm's performance.

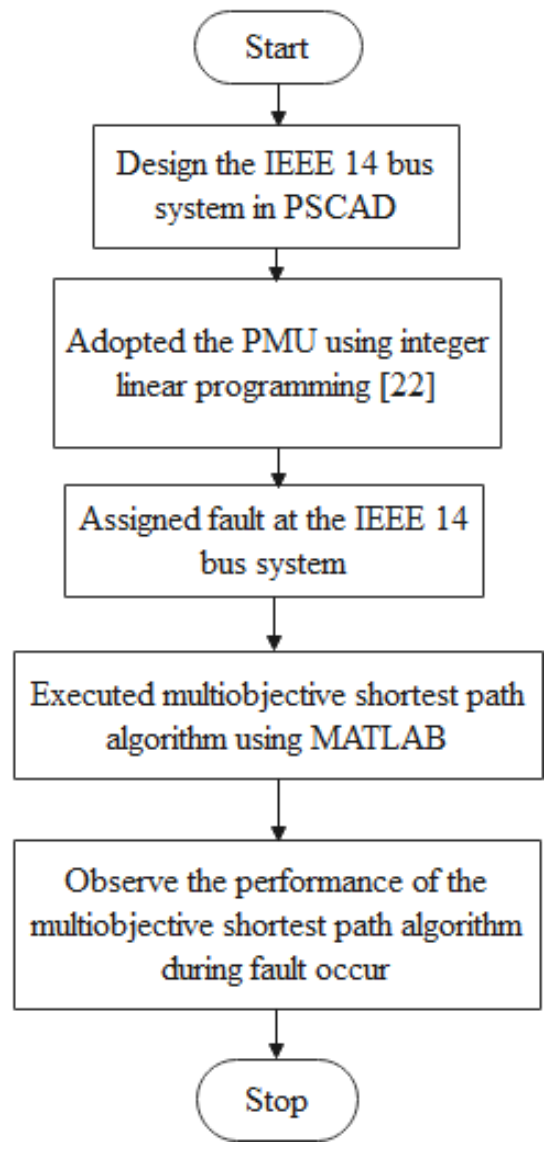

Fig. 2. Flowchart.

TABLE I. LIST OF FAULT LOCATION

\begin{tabular}{|l|l|l|l|}
\hline No. Fault & location & Fault & Location \\
\hline 1 & Bus 9 to 10 & 11 & Bus 7 to 9 \\
\hline 2 & Bus 10 to 11 & 12 & Bus 4 to 9 \\
\hline 3 & Bus 6 to 11 & 13 & Bus 5 to 6 \\
\hline 4 & Bus 6 to 13 & 14 & Bus 1 to 5 \\
\hline 5 & Bus 6 to 12 & 15 & Bus 1 to 2 \\
\hline 6 & Bus 12 to 13 & 16 & Bus 5 to 2 \\
\hline 7 & Bus 13 to 14 & 17 & Bus 2 to 4 \\
\hline 8 & Bus 9 to 10 & 18 & Bus 2 to 3 \\
\hline 9 & Bus 4 to 9 & 19 & Bus 3 to 4 \\
\hline 10 & Bus 4 to 7 & \multicolumn{2}{|l}{} \\
\cline { 1 - 2 } & &
\end{tabular}

\section{RESUlt AND Discussion}

In the IEEE-14 bus system, 19 faults were assigned at different locations and tested for data collection based on the utilisation of multi objective shortest route algorithm. Each fault was then simulated using a multi-objective shortest path method in MATLAB to determine the shortest path length. All of the data in this section was collected by simulation, and was evaluated by plotting graphs for further discussion. 


\section{A. Fault Simulation on Section}

The IEEE-14 bus system was used to simulate 19 fault scenarios. Each type of fault was simulated between buses and different locations. Table II shows the details of the fault simulation point. Based on the table, the fault point was simulated in each section (between bus to bus). For example, fault 1 was simulated between buses 9 and 10, with a distance of 18.9 kilometers between them. All the fault points were simulated in the middle of the section.

\section{B. Path Analysis}

Path analysis is discussed in this section with different fault simulation points considered. Every fault point will be analyzed, with detailed results and explanations. For each fault case, the result will be compared to the normal path and the new path. Before the fault occurs, the multi-objective shortest path algorithm calculates the normal path distance. Meanwhile, during the fault, the multi-objective shortest path algorithm calculates the new path distance. There were six paths that connect the buses for each fault case.

Table III shows the route taken to connect to other buses, as well as the distance used to calculate fault 1 . The first fault occurs in the middle of the section between buses 9 and 10. If the fault occurs at the path connecting the phasor measurement unit and the buses, the path should be changed. The path distance is referred to as the main route in normal conditions (without fault), as shown in Table III.

TABLE II. FAULT POINT

\begin{tabular}{|l|l|l|l|}
\hline \multicolumn{4}{|c}{ TABLE II. FAULT POINT } \\
\hline No. & Fault Location & $\begin{array}{l}\text { Distance between } \\
\text { bus (Km) }\end{array}$ & $\begin{array}{l}\text { Distance of Fault } \\
\text { (Km) }\end{array}$ \\
\hline 1 & Bus 9 to 10 & 18.9 & 9.45 \\
\hline 2 & Bus 10 to 11 & 42.9 & 21.45 \\
\hline 3 & Bus 6 to 11 & 44.4 & 22.2 \\
\hline 4 & Bus 6 to 13 & 29.1 & 14.55 \\
\hline 5 & Bus 6 to 12 & 57.1 & 28.55 \\
\hline 6 & Bus 12 to 13 & 44.7 & 22.35 \\
\hline 7 & Bus 13 to 14 & 77.8 & 38.9 \\
\hline 8 & Bus 9 to 14 & 60.4 & 30.2 \\
\hline 9 & Bus 4 to 9 & 124 & 62 \\
\hline 10 & Bus 4 to 7 & 46.7 & 23.35 \\
\hline 11 & Bus 7 to 9 & 24.6 & 12.3 \\
\hline 12 & Bus 4 to 5 & 9.4 & 4.7 \\
\hline 13 & Bus 5 to 6 & 56.3 & 28.15 \\
\hline 14 & Bus 1 to 5 & 24.9 & 24.9 \\
\hline 15 & Bus 1 to 2 & 13.2 & 6.6 \\
\hline 16 & Bus 2 to 5 & 38.9 & 19.45 \\
\hline 17 & Bus 2 to 4 & 39.4 & 19.7 \\
\hline 18 & Bus 2 to 3 & 44.2 & 22.1 \\
\hline 19 & Bus 3 to 4 & 38.2 & 19.1 \\
\hline
\end{tabular}

TABLE III. PATH DISTANCE FOR FAULT 1

\begin{tabular}{|l|l|l|l|l|}
\hline Route & Main route & $\begin{array}{l}\text { Normal path } \\
\text { (Km) }\end{array}$ & $\begin{array}{l}\text { Route } \text { after } \\
\text { fault }\end{array}$ & $\begin{array}{l}\text { New } \\
\text { path } \\
\text { (Km) }\end{array}$ \\
\hline 1 & 5 to 2 & 38.9 & 5 to 2 & 38.9 \\
\hline 2 & 5 to 6 & 56.3 & 5 to 6 & 56.3 \\
\hline 3 & 5 to 4 to 7 to 9 & 80.7 & 5 to 4 to 7 to 9 & 80.7 \\
\hline 4 & 6 to 5 to 2 & 95.2 & 6 to 5 to 2 & 95.2 \\
\hline 5 & 6 to 11 to 10 to 9 & 160.2 & $\begin{array}{l}6 \text { to } 5 \text { to } 4 \text { to } 7 \\
\text { to } 9\end{array}$ & 137 \\
\hline 6 & 2 to 4 to 7 to 9 & 110.7 & 2 to 4 to 7 to 9 & 110.7 \\
\hline
\end{tabular}

Table III is made up of five columns. The number of the route appears first, followed by the main route (normal condition of the route). The distance for the normal path is represented in the third column. The fourth column represents the route after a fault, and the last column represents the new path's distance. According to Table III, the distance between bus 5 and bus 2 for route 1 is 39.8 kilometers. When fault 1 occurred, no change was observed in the route condition and distance. Change in route number 5 , which is the main route path, can be seen starting from bus 6 , continuing to bus 11 , bus 10 , and bus 9 . The simulated result for the main path was 106.2 kilometers. Based on the observation, the new path distance was recorded at 137 kilometers. This value differs from the normal path by 30.8 kilometers. It means that the multi-objective method for determining the new path of the distance has a big impact on path distance effectiveness. Route 5 demonstrated a different distance between the main route and the route after the fault occurs, as shown in Fig. 3. The normal distance for Route 5 was 106.2 kilometers, but after the fault, the distance increased to 137 kilometers. This is due to the fact that the fault 1 occurs between buses 10 and 11 . The algorithm must calculate the shortest path to connect buses 6 and 9 since the main route used is between bus 10 and 11. The algorithm looked at every possible path and chose the shortest one. The path for route 5 was made up of buses 6,5 , 4,7 , and 9 . Since the fault does not interrupt the path, the other route is unaffected.

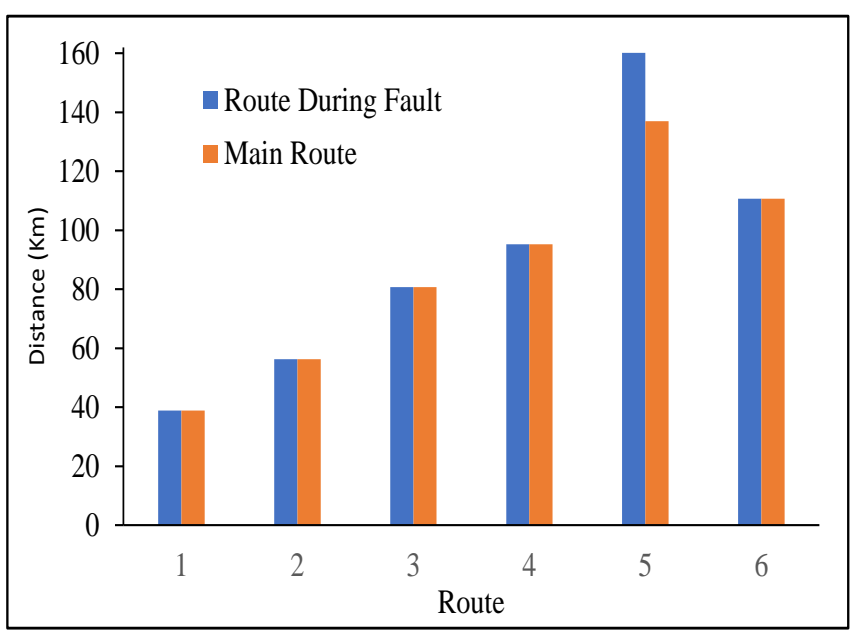

Fig. 3. Distance against Route for Fault 1. 


\section{Distance Comparison between every Fault for each Route}

Distance comparison is discussed in this section for each fault case on each route. An example of a distance comparison between each route's faults is presented in Table IV. The condition of the phasor measurement unit at bus 2 and the center at bus 5 is represented by Route 1 .

When fault 1 occurs between buses 9 and 10, it resulted in a path distance of 38.9 kilometers. Except for the distance for fault case 16, all of the distances for route 1 are the same. Since the fault occurs between bus 2 to bus 5 , the distance for fault case 16 is 48.8 kilometers. As a result, the path from bus 5 to bus 2 cannot be used, and the multi-objective shortest path algorithm must find a new shortest path. Other faults were unaffected because the path that connects bus 5 and bus 2 was not interrupted.

TABLE IV. DISTANCE FOR ROUTE 1

\begin{tabular}{|l|l|l|l|}
\hline No. of fault & Distance & No. of fault & Distance \\
\hline 1 & $38.9 \mathrm{Km}$ & 11 & $38.9 \mathrm{Km}$ \\
\hline 2 & $38.9 \mathrm{Km}$ & 12 & $38.9 \mathrm{Km}$ \\
\hline 3 & $38.9 \mathrm{Km}$ & 13 & $38.9 \mathrm{Km}$ \\
\hline 4 & $38.9 \mathrm{Km}$ & 14 & $38.9 \mathrm{Km}$ \\
\hline 5 & $38.9 \mathrm{Km}$ & 15 & $38.9 \mathrm{Km}$ \\
\hline 6 & $38.9 \mathrm{Km}$ & 16 & $48.8 \mathrm{Km}$ \\
\hline 7 & $38.9 \mathrm{Km}$ & 17 & $38.9 \mathrm{Km}$ \\
\hline 8 & $38.9 \mathrm{Km}$ & 18 & $38.9 \mathrm{Km}$ \\
\hline 9 & $38.9 \mathrm{Km}$ & 19 & $38.9 \mathrm{Km}$ \\
\cline { 1 - 3 } 10 & $38.9 \mathrm{Km}$ & \multicolumn{2}{|l}{} \\
\cline { 1 - 3 } & \multicolumn{2}{|l}{} &
\end{tabular}

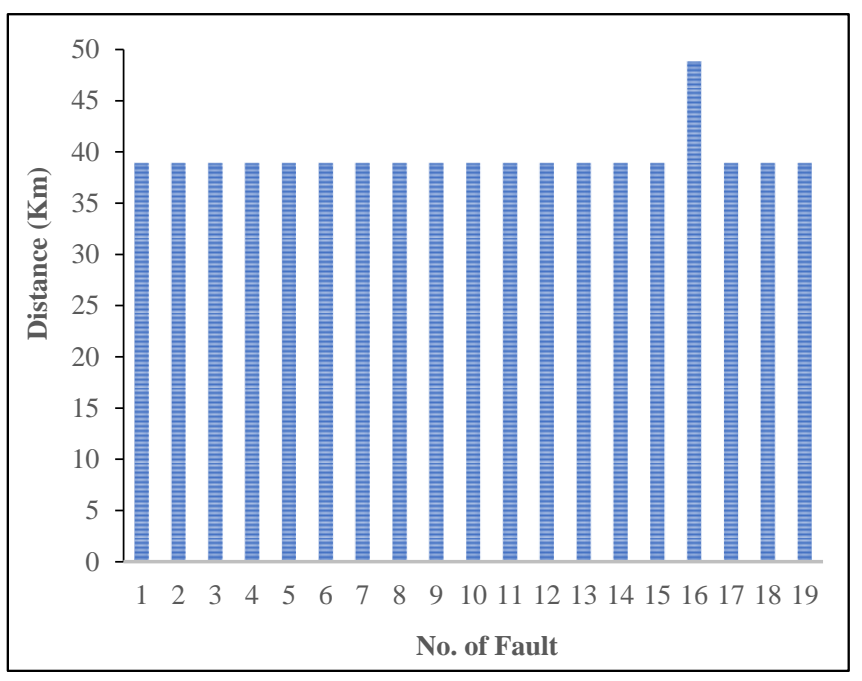

Fig. 4. Distance against Fault for Route 1.

Fig. 4 depicts the distance between bus 5 and bus 2 during a fault occurrence. On the IEEE-14 bus system, 19 different faults were assigned. Except for fault number 16, every fault case takes the same path to connect bus 5 to bus 2 and the path distance recorded is 38.9 kilometers. As the fault happens between buses 5 and 2, the algorithm calculates various paths for fault 16. Although bus 2 is another available option of path, the algorithm needs to choose different path since the path between bus 5 and 2 cannot be used. Therefore, the algorithm then assigns another path, which is between bus 5 to 4.

\section{CONCLUSION}

This work presents a new method based on multi-objective shortest path algorithm for determining a new path distance of connected buses when faults occur at various locations within a test system. The IEEE-14 bus system was tested with nineteen different types of faults to investigate the performance of the proposed algorithm during the fault events. The path distances were measured and the shortest path for every fault case was then determined. Based on the results, it was found that the shortest path of the buses connected to each other is significantly influenced by the location of the fault. In the event of a fault, the proposed algorithm will choose a new path based on the shortest distance, excluding the old route, which is already affected by the fault. Based on the obtained results, the fault occurrences have significantly affected the main route consisting of routes $6,11,10$ and 9 . It can be concluded that the multiobjective shortest path algorithm is capable of estimating the possibility of new path distance during a fault in the IEEE 14-bus system.

\section{ACKNOWLEDGEMENT}

The authors thanks to the Airlangga University, Indonesia and 2021 SATU Joint Research Scheme (JRS) no UM67.

REFERENCES

[1] Venkatasubramanian, Vaithianathan, et al. "Wide-area monitoring and control algorithms for large power systems using synchrophasors." 2009 IEEE/PES Power Systems Conference and Exposition. IEEE, 2009.

[2] Gore, Rahul, and Mallikarjun Kande. "Analysis of wide area monitoring system architectures." 2015 IEEE International Conference on Industrial Technology (ICIT). IEEE, 2015.

[3] Bose, Anjan. "Smart transmission grid applications and their supporting infrastructure." IEEE transactions on Smart Grid 1.1 (2010): 11-19.

[4] Chen, Jian, and Ali Abur. "Placement of PMUs to enable bad data detection in state estimation." IEEE Transactions on Power Systems 21.4 (2006): 1608-1615.

[5] Nuqui, Reynaldo F., and Arun G. Phadke. "Phasor measurement unit placement techniques for complete and incomplete observability." IEEE Transactions on Power Delivery 20.4 (2005): 2381-2388.

[6] Xu, Bei, and Ali Abur. "Observability analysis and measurement placement for systems with PMUs." IEEE PES Power Systems Conference and Exposition, 2004.. IEEE, 2004.

[7] Almunif, Anas, and Lingling Fan. "Mixed integer linear programming and nonlinear programming for optimal PMU placement." 2017 North American Power Symposium (NAPS). IEEE, 2017.

[8] Li, Yikui, Jie Li, and Lei Wu. "A Novel Integer Linear Programming Based Optimal PMU Placement Model." 2018 North American Power Symposium (NAPS). IEEE, 2018.

[9] Ahmed, Muhammad Musadiq, and Kashif Imran. "An Optimal PMU Placement Against N-1 Contingency of PMU Using Integer Linear Programming Approach." 2019 International Conference on Applied and Engineering Mathematics (ICAEM). IEEE, 2019.

[10] Reddy, K. SivaRama Krishna, et al. "Implementation of Integer Linear Programming and Exhaustive Search algorithms for optimal PMU placement under various conditions." 2015 IEEE Power, Communication and Information Technology Conference (PCITC). IEEE, 2015. 
[11] Gou, B. (2008). Optimal placement of PMUs by integer linear programming. IEEE Transactions on power systems, 23(3), 1525-1526.

[12] Chakrabarti, S., \& Kyriakides, E. (2008). Optimal placement of phasor measurement units for power system observability. IEEE Transactions on power systems, 23(3), 1433-1440.

[13] Bashian, Amir, et al. "Optimal design of a wide area measurement system using hybrid wireless sensors and phasor measurement units." Electronics 8.10 (2019): 1085.

[14] Singh, Satyendra P., and Shiv P. Singh. "Optimal cost wide area measurement system incorporating communication infrastructure." IET Generation, Transmission \& Distribution 11.11 (2017): 2814-2821.

[15] James, J. Q., et al. "A unified framework for wide area measurement system planning." International Journal of Electrical Power \& Energy Systems 96 (2018): 43-51.

[16] Martin, Kenneth E. "Synchrophasor measurements under the IEEE standard C37. 118.1-2011 with amendment C37. 118.1 a." IEEE Transactions on Power Delivery 30.3 (2015): 1514-1522.

[17] De La Ree, Jaime, et al. "Synchronized phasor measurement applications in power systems." IEEE Transactions on smart grid 1.1 (2010): 20-27.
[18] Phadke, Arun G., and James S. Thorp. Synchronized phasor measurements and their applications. Vol. 1. New York: Springer, 2008.

[19] Balu, Neal J., Mark G. Lauby, and P. Kundur. "Power system stability and control." Electrical Power Research Institute, McGraw-Hill Professional (1994).

[20] Rashidi, Farzan, et al. "Optimal placement of PMUs with limited number of channels for complete topological observability of power systems under various contingencies." International Journal of Electrical Power \& Energy Systems 67 (2015): 125-137.

[21] Abiri, Ebrahim, Farzan Rashidi, and Taher Niknam. "An optimal PMU placement method for power system observability under various contingencies." International transactions on electrical energy systems 25.4 (2015): 589-606.

[22] Ghasemkhani, A., Monsef, H., Rahimi-Kian, A., \& Anvari-Moghaddam, A. (2017). Optimal design of a wide area measurement system for improvement of power network monitoring using a dynamic multiobjective shortest path algorithm. IEEE Systems Journal, 11(4), 2303-2314. 